\title{
Commentary: Novel Use of Offset Analgesia to Assess Adolescents and Adults with Treatment Resistant Endometriosis-Associated Pain
}

This article was published in the following Dove Press journal: Journal of Pain Research

\author{
Claire E Lunde ${ }^{1-3, *}$ \\ Edina Szabo ${ }^{1,4, *}$ \\ Scott A Holmes ${ }^{1,4}$ \\ David Borsook ${ }^{1,4}$ \\ Christine B Sieberg (iD) 1,2,5 \\ 'Center for Pain and the Brain (P.A.I.N. \\ Group), Department of Anesthesiology, \\ Critical Care, and Pain Medicine, Boston \\ Children's Hospital, Boston, MA, USA; \\ ${ }^{2}$ Biobehavioral Pediatric Pain Lab, \\ Department of Psychiatry and Behavioral \\ Sciences, Boston Children's Hospital, \\ Boston, MA, USA; ${ }^{3}$ Nuffield Department \\ of Women's and Reproductive Health, \\ Medical Sciences Division, University of \\ Oxford, Oxford, Oxfordshire, UK; \\ ${ }^{4}$ Department of Anesthesiology, Harvard \\ Medical School, Boston, MA, USA; \\ ${ }^{5}$ Department of Psychiatry, Harvard \\ Medical School, Boston, MA, USA \\ *These authors contributed equally to \\ this work
}

Correspondence: Christine B Sieberg Biobehavioral Pediatric Pain Lab, Department of Psychiatry and Behavioral Sciences, Boston Children's Hospital, 21 Autumn St, Boston, MA, USA

Tel + | 617-355-2296

Email christine.sieberg@childrens. harvard.edu
Background and Objective: Endometriosis, affecting approximately 176 million adults and adolescents worldwide, is a debilitating condition in which uterine tissue grows outside the uterus. The condition costs the US economy approximately $\$ 78$ billion annually in painrelated disability. By understanding the neural underpinnings of endometriosis-associated pain (EAP) and risk factors for chronification, translational research methods could lessen diagnostic delays and maximize successful pain remediation. This can be accomplished by the novel use of a known method, offset analgesia (OA), to better elucidate the neural mechanisms that may contribute to and maintain EAP. This commentary will provide justification and rationale for the use of OA in the study of EAP.

Conclusion: Utilizing an OA paradigm in patients with endometriosis, especially adolescents, may (1) provide insight into neural mechanisms contributing to pain maintenance, which could capture those at-risk for the transition to chronic pelvic pain, (2) provide a metric for the development of future centrally mediated treatment options for this population, and (3) elucidate the brain changes that result in resistance to treatment and pain chronification.

Keywords: endometriosis, chronic pain, offset analgesia, central nervous system

\section{Endometriosis and Pain}

Endometriosis, a debilitating condition in which uterine tissue grows outside the uterus, affects approximately 176 million adults and adolescents worldwide ${ }^{1}$ and costs the US economy approximately $\$ 78$ billion annually in pain-related disability. ${ }^{2}$ It has a deleterious impact on physical and emotional functioning, as well as quality of life, and is the leading cause of chronic pelvic pain (CPP). ${ }^{3}$ Following laparoscopic surgery, to both confirm the diagnosis and remove the ectopic tissue, patients are commonly managed with a hormonal regimen during the childbearing years. Despite these treatments, approximately $30 \%$ of women report no improvement in pain after surgery and many other patients report frequently recurring endometriosis-associated pain (EAP) without evidence of recurrent disease. ${ }^{4}$ With this high prevalence of pain and disease burden, there is a lack of effective treatments for this subset of treatment nonresponders who continue to experience EAP. ${ }^{5}$ Endometriosis in adolescents is an even more challenging problem as it may present with a number of clinical and pathological differences that are not observed in adult women. Nevertheless, given the chronicity of the disease, the challenge is to avoid a delay in diagnosis, understand 
the disease, and direct effective therapies at an early age. ${ }^{6}$ Small pilot studies ${ }^{7,8}$ in adult women have shown the maintenance and chronicity of EAP to be associated with structural and functional alterations in regions related to pain modulation suggesting that EAP is not explained by endometriosis alone, but alternatively may be explained by dysfunction in the Central Nervous System (CNS) either as a defective pain modulatory system or an increase in neuronal responsivity in central sensory and emotional pain pathways. Recent animal models have shown that the type of nerve that innervates the endometriosis lesion, as well as the location of the lesion, impact the CNS differently and thus dictate how the pain is modulated, such as peripherally, centrally, and by other central dynamic processes. ${ }^{9}$ As the onset of this condition may begin before menarche $e^{10,11}$ and adult women report their symptoms beginning during adolescence, ${ }^{12}$ addressing early pre-symptomatic markers of pain chronification in the pediatric brain may change the prognosis by increasing the quality of life and protecting the health and reproductive system of adolescents with endometriosis. This can be accomplished by the novel use of a known method to better elucidate the neural mechanisms that may contribute to and maintain EAP - offset analgesia (OA). OA is increasingly used to measure endogenous pain inhibition, which is considered one of the central mechanisms facilitating (or preventing) pain and contributing to pain chronification.

\section{Peripheral or Central Processing of Pain}

One main reason for the lack of effective treatments is a poor understanding of the neural mechanisms contributing to the maintenance and exacerbation of pain in this population. Historically, the pain associated with endometriosis has been conceptualized and treated peripherally (eg, surgical excision of the lesion, oral contraceptives, NSAIDs, etc.) resulting from inflammatory nociceptive molecules or damaged nerves, leaving the role of the CNS largely ignored. ${ }^{6}$ Studies exploring changes in the CNS have found increased sensitivity to pain within and outside of the areas of the pelvis among endometriosis patients, as well as decreased gray matter volume (GMV) and increased functional connectivity in brain regions involved in pain processing such as the medial prefrontal cortex, anterior insula, cingulate gyrus, thalamus and putamen. ${ }^{7,8}$ In line with this, endometriosis patients without CPP did not show hyperalgesia and reduced GMV, but they exhibited increased GMV in the periaqueductal gray (PAG) which is an important structure in the endogenous pain modulation system. ${ }^{8}$ Such findings support the notion that the alterations in the central processing of pain likely contribute to $\mathrm{CPP}$ in endometriosis.

\section{Offset Analgesia in Experimental Settings \\ Overview - Why OA?}

The most frequently used assessment paradigms for capturing this inhibitory pain mechanism are conditioned pain modulation (CPM) and OA. While CPM measures spatial filtering of pain perception (eg, the pain induced by one noxious stimulus is inhibited by another noxious stimulus applied to a remote area of the body), OA is related to filtering nociceptive information in the temporal domain. ${ }^{13}$ OA was first defined by Grill and Coghill ${ }^{14}$ as a form of endogenous pain inhibition characterized by a disproportionately large reduction in pain perception after a small decrease in temperature during noxious thermal stimulation. Although it has been proposed that both OA and CPM are mediated by central and peripheral mechanisms, ${ }^{15} \mathrm{OA}$ seems to be related to increased activation in brain regions involved in pain modulation (eg, PAG, dorsolateral prefrontal cortex, anterior insula, brainstem), whereas CPM induces activity reduction in regions connected to afferent nociceptive processing which might implicate more brain-derived pain modulation during $\mathrm{OA} .{ }^{13} \mathrm{OA}$ is increasingly used to measure endogenous pain inhibition because when compared to CPM, it applies a noxious stimulus that evokes only moderate pain and the duration of the painful stimulus is also shorter which makes the paradigm more suitable for patients, particularly for adolescents. ${ }^{15}$ In addition, OA responses are not affected by centrally-acting drugs, while in some studies, CPM effects appear to diminish considerably after taking these medications. ${ }^{15}$ Hence, OA occurs predominantly by an opioid-independent mechanism which emphasizes the role of other neurotransmitters involved in the modulation of pain measured by this paradigm. ${ }^{16}$ Notably, some of these studies also reported that $\mathrm{OA}$ responses could not be modified in patients with chronic pain by analgesics such as ketamine,${ }^{17}$ morphine vs placebo, ${ }^{17}$ NSAIDs and acetaminophen, ${ }^{18}$ tapentadol, ${ }^{19}$ and hydromorphone. ${ }^{20}$

Human studies of EAP as a centrally mediating phenomenon, especially in adolescents, are lacking. Instead, 
Quantitative Sensory Testing (QST), ${ }^{21}$ a set of psychophysical methods assessing pain sensitivity and sensory nerves, to produce measures of ongoing CNS and Peripheral Nervous System sensitization, has been the main focus in the field. General chronic pain, ${ }^{22}$ complex regional pain syndrome, ${ }^{23}$ migraines, ${ }^{24}$ abdominal pain, ${ }^{25}$ sickle cell disease, ${ }^{26,27}$ fibromyalgia, ${ }^{28}$ juvenile idiopathic arthritis, ${ }^{29}$ and cerebral palsy, ${ }^{30}$ have been the focus of adolescent QST studies, which all suggest that pediatric chronic pain can lead to maladaptive peripheral and central sensory processing. As more recent findings have suggested $^{31}$ the central effects of EAP should be further explored and the use of OA could provide insight to the underlying neural mechanisms and persistence of CPP.

\section{The OA Paradigm}

Many OA paradigms have been described (see reviews ${ }^{15,32}$ on multiple paradigms); however, $\mathrm{OA}$ in the magnetic resonance imaging (MRI) setting, ${ }^{33-36}$ typically uses the 3 -temperature paradigm (Figure 1$)^{14}$ defined by a rating of 5 out of 10 on a visual analog scale (VAS). Notably, this standard 3-temperature paradigm has not been tested in a MRI setting with a pediatric population; however, OA was assessed in youth with chronic pain one time (outside MRI), applying an individualized noxious thermal stimulus of 50/100 on a VAS. ${ }^{37}$ Using a thermal stimulator which is placed in contact with the skin, a preset computer-controlled temperature paradigm delivers the specific temperature pattern with the subject rating their pain in real time. A response to this dynamic test stimulus or offset trial (Figure 1A) consists of a reduction in self-reported pain intensity when the test temperature is applied for 5 seconds, raised by $1{ }^{\circ} \mathrm{C}$ for 5 seconds, reduced by $1^{\circ} \mathrm{C}$, and held for 20 seconds. The response to a constant test stimulus (Figure 1B) on the skin consists of a reduction in self-reported pain intensity when the test temperature is applied at a constant rate for 30 seconds. Lastly, the response to a controlled stimulus (Figure 1C) on the skin consists of a reduction in self-reported pain intensity when the test temperature is applied for $5 \mathrm{sec}-$ onds, reduced to $32^{\circ} \mathrm{C}$, and held for 30 seconds. One common method for quantifying the magnitude of $\mathrm{OA}$ is by determining the percentage of difference between the highest VAS scores during the second temperature and the lowest VAS scores during the third temperature. However, as OA responses can be partially due to adaptation effects, including the constant temperature trial is recommended to calculate OA effects. Accounting for the time-dependent changes might mitigate overestimating the magnitude of OA. ${ }^{14}$ Functional MRI (fMRI) studies also used the constant thermal trial to evaluate OA responses in terms of pain adaptation and habituation. ${ }^{36,38}$ In the study of Zhang et $\mathrm{al},{ }^{36}$ the constant stimulus trials served as control blocks to investigate brain activations to the attenuated $\mathrm{OA}$ effects in patients with various chronic pain disorders. Compared to healthy controls, reduced brain activation was found in regions associated with the descending pain modulatory, such as the anterior cingulate cortex, dorsolateral prefrontal cortex, and reward systems, such as the medial prefrontal cortex, putamen, nucleus accumbens.

\section{Offset Analgesia as a Clinical Tool}

Recent studies report decreased or absent OA responses in patients with fibromyalgia, ${ }^{38}$ neuropathic pain, ${ }^{17}$ migraine, ${ }^{39}$ and chronic pain of various etiologies. ${ }^{36,37,40}$ In addition, three studies focusing only on chronic patients indicated decreased OA responses in patients with diabetic polyneuropathy, knee osteoarthritis, ${ }^{19}$ and chronic radicular pain ${ }^{17,18,20}$ although there was large interindividual variation. In patients with chronic pain, the attenuation of OA effects was observed by delayed offset and a relatively minor decrease in pain scores following the $1^{\circ} \mathrm{C}$ decrease in temperature as opposed to the disproportionately large reduction in pain perception among healthy controls (Figure 1). The body of literature on OA does provide strong evidence that attenuated OA responses are consistently found in patients with chronic pain, indicating a lack of ability to modulate changes in pain perception. Alterations of central pain inhibitory and reward systems might be associated with pain chronification, and the magnitude of OA could be a useful index to distinguish the $30 \%$ of patients with endometriosis who have persistent EAP after treatment.

\section{Offset Analgesia in Endometriosis}

Although OA responses have been found to be attenuated in patients with various chronic pain conditions, it is unknown in EAP. Only two studies have used CPM on patients with $\mathrm{EAP}^{41}$ and patients with dysmenorrhea ${ }^{42}$ (common symptom and predictor of endometriosis) which reported inconsistent results, calling for further investigation within this population. Furthermore, less efficient CPM was reported in comparable disorders such as irritable bowel syndrome ${ }^{43}$ or CPP syndrome, ${ }^{44}$ but not in premenstrual dysphoric disorder. ${ }^{45}$ It should be also noted that the role of female-specific reproductive hormones on 


\section{A. Offset Trial: Magnitude of OA}
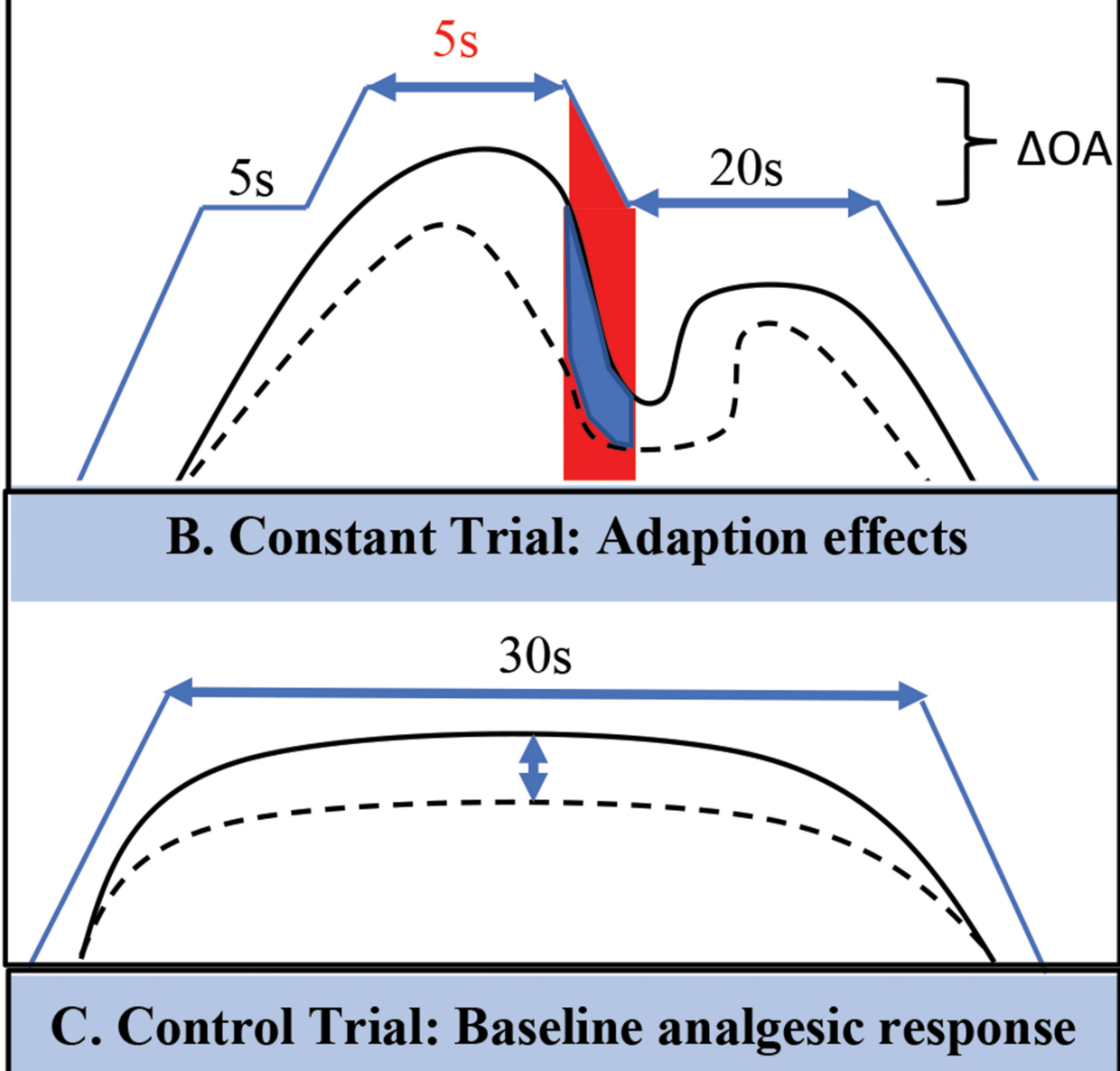

$5 \mathrm{~s}$

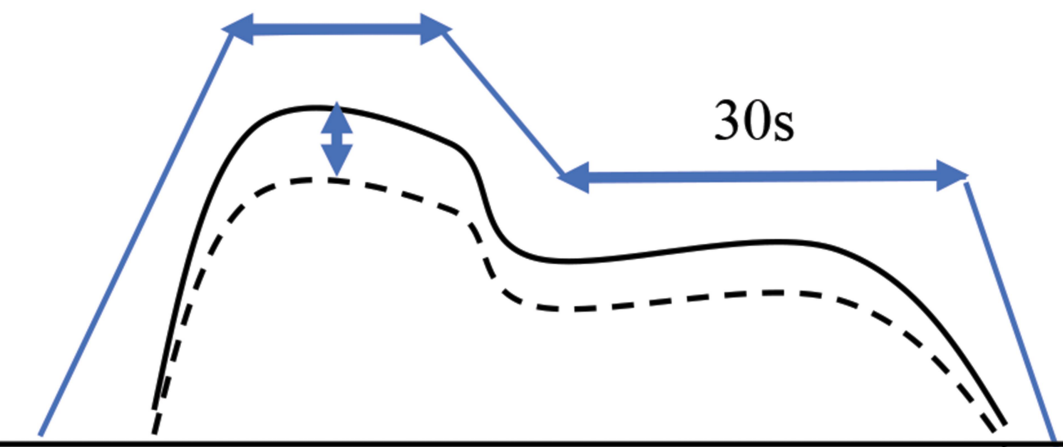

Figure I A commonly used 3-temperature OA paradigm includes Offset, Constant and Control trials. The $x$-axis represents time (seconds), with the $y$-axis representing temperature (Celsius). Participants continuously rate their pain using a visual analog scale (VAS) of 0-100. (A) The Offset Trial consists of individualized temperature 50/I00 VAS for $5 \mathrm{~s}$, with a $10 \mathrm{C}$ increase for $5 \mathrm{~s}$, and ending with a decrease in 10C for 20s. (B) The Constant Trial consists of 30s at 20C below the 50/100 VAS. (C) The Control Trial consists of $5 \mathrm{~s}$ at 50/100 VAS and then 30s at 320C. A 40s interval between each test is suggested. The redline below highlights the Offset Analgesia: a small decrease (IOC) in temperature during noxious thermal stimulation which leads to a disproportionately large reduction in pain perception. One method for measuring the magnitude of OA is determining the percentage of difference between the highest VAS scores during the second temperature (VASmax 5s) and the lowest VAS scores during the third temperature (VASmin 20s) in the Offset Trial: $\triangle O A=V A S m a x ~ 5 s-V A S m i n$ 20s. Including a Constant Trial is recommended for calculating the OA magnitude to rule out adaptation effects. This OA paradigm captures endogenous pain inhibition, and it is associated with decreased brain activation of the descending pain modulatory and reward systems in patients with chronic pain. 
sensation and perception is not fully understood, although some studies have found that the menstrual cycle influences pain sensations elicited by noxious stimuli. ${ }^{46-49}$ In line with this, OA has been found to be somatotopically organized in episodic migraine, a cyclic disease where pain perception changes according to the migraine phase. ${ }^{39}$ Specifically, OA responses were measured at the forehead and forearm during the headache-free period, and the impaired pain inhibition was restricted to the affected area (the head). Based on these results, measuring OA on the lower abdomen (besides the forearm, which is the most commonly used examination site) should be considered in patients with EAP.

With ongoing pain (intermittent or constant), alterations in the sensitivity/responsivity of neural networks encompass those beyond the classic sensory pathways (eg, spinothalamic tract) and involve anxiety, cognition, memory, and the normal function of the brain's pain inhibitory (descending modulation) systems are thought to take place. ${ }^{50}$ Through these changes in neural networks, one measurable process is a change in descending pain inhibition, which can be evaluated using the described OA paradigm. Given that EAP is a multifaceted and complex problem, there is a desperate need for a new approach to understand the neural mechanisms in both adult and pediatric cases of endometriosis. This could be accomplished by using translational research methods, such as $\mathrm{OA}$, to determine if the brain regions associated with pain evolution and maintenance have been altered, leading to increased pain sensitivity which in turn could lead to an earlier finding of a more effective treatment. Based on the aforementioned results, the diminished endogenous pain inhibition measured with OA would thus be a reflection of an altered brain system in which there is an increased sensitivity to pain and increased resistance to treatment in conditions such as CPP associated with endometriosis.

A better understanding of the neural mechanisms occurring in the CNS and of the role of the brain's inhibitory response system in the development, maintenance, and exacerbation of EAP would provide a necessary paradigm shift for the field of adolescent and adult female reproductive health. Specifically, assessing the OA response in this patient population would: (1) enhance our understanding of the structural and functional alterations in the CNS pain regulatory system due to CPP in patients with endometriosis; (2) provide a measurable change in the brain's structure to follow such patients in the clinic; (3) potentially provide a measurable change in the brain for those who will develop EAP; and (4) define an initial paradigm that may enhance our capability for developing individually tailored patient-oriented interventions.

\section{Paradigm Shift for the Treatment of EAP}

Evaluating OA responses could be beneficial in a clinical setting because it would give further information about the patient responses to different interventions. There have only been a few studies assessing the role of pharmacological interventions on the OA response and indicate that while the magnitude of OA was not affected by different analgesics, the reported clinical pain intensity decreased after treatment, ${ }^{17,18,20}$ indicating that pain relief may not necessarily be associated with the normalization of the central pain inhibiting system. Other centrally targeted therapies, such as Cognitive Behavioral Therapy, have largely been unexplored as they relate to the OA response. Patients with endometriosis and CPP appear to be a heterogeneous group with different levels of peripheral and central sensitization. Thus, some patients are likely to react better to therapies involving pharmacologic and cognitive-behavioral approaches that focus on the central aspect of pain than to repeated surgeries aimed at treating endometriosis. The OA paradigm might provide a useful tool to predict or confirm treatment responders vs nonresponders and to reveal the extent to which different treatments can contribute to reversing the central changes.

\section{Conclusion}

By understanding the neural underpinnings of endometriosis-associated pain and risk factors for chronification, translational research methods, such as OA, could lessen diagnostic delays and maximize successful pain remediation. As OA has been administered at the volar side of the arm and the same time intervals (5 s, $5 \mathrm{~s}, 20 \mathrm{~s})$ for many studies, this can be the recommended procedure for future studies of adolescents and women with treatment resistant endometriosis-associated EAP. Additionally, using the lower abdomen as an examination site should be considered. There is great need for estimating OA in patients with endometriosis, especially adolescents, as the way forward because it can: (1) provide insight into neural mechanisms contributing to pain maintenance, which could capture those at-risk for the transition to CPP, (2) provide a metric for the development of future centrally mediated treatment options for this population, and (3) 
elucidate the brain changes that result in resistance to treatment and pain chronification. This novel use of an existing tool is of paramount importance due to the reduced quality of life in young patients and for the known grim condition outcomes in adulthood. To date, no previous studies have explored OA in adult or pediatric patients with EAP and given that most patients report symptoms of EAP during adolescence, this would be a critical time to investigate pain modulation with the goal of decreasing pain in women with endometriosis. More research in understanding the underlying neural mechanisms and centralized effects of pain within adolescents and adults with endometriosis is of great importance and the proposed utilization of $\mathrm{OA}$ in this population will hopefully move the field of reproductive health forward.

\section{Author Contributions}

ES and CEL conducted the literature review. SAH, CBS, and DB contributed to manuscript preparation. All authors made a significant contribution to the work reported, whether that is in the conception, and took part in drafting, revising or critically reviewing the article; gave final approval of the version to be published; have agreed on the journal to which the article has been submitted; and agree to be accountable for all aspects of the work. Ms. Lunde and Dr. Szabo both equally contributed to first authorship of this manuscript.

\section{Funding}

CBS and DB are Partnering PIs on a Peer Review Medical Research Program Investigator-Initiated Research Award from the United States Department of Defense (W81XWH19105060); CBS is also funded by a K23 Career Development Award from NIH (GM123372).

\section{Disclosure}

David Borsook reports personal fees from Redpin, outside the submitted work. The authors have no other potential conflicts of interest to disclose.

\section{References}

1. Simoens S, Dunselman G, Dirksen C, et al. The burden of endometriosis: costs and quality of life of women with endometriosis and treated in referral centres-of-illness/quality of life/international/multi-centre. Hum Reprod. 2012;27(5):1292-1299. doi:10.1093/humrep/ $\operatorname{des} 073$

2. Soliman AM, Surrey E, Bonafede M, Nelson JK, Castelli-Haley J. Real-world evaluation of direct and indirect economic burden among endometriosis patients in the United States. Adv Ther. 2018;35 (3):408-423. doi:10.1007/s12325-018-0667-3
3. Bourdel N, Chauvet P, Billone V, et al. Systematic review of quality of life measures in patients with endometriosis. PLoS One. 2019;14 (1). doi:10.1371/journal.pone. 0208464

4. Abbott J, Hawe J, Hunter D, Holmes M, Finn P, Garry R. Laparoscopic excision of endometriosis: A randomized, placebo-controlled trial. Fertil Steril. 2004;82(4):878-884. doi:10.1016/j.fertnstert.2004.03.046

5. Lamvu G, Soliman AM, Manthena SR, Gordon K, Knight J, Taylor HS. Patterns of prescription opioid use in women with endometriosis: evaluating prolonged use, daily dose, and concomitant use with benzodiazepines. Obstet Gynecol. 2019;133(6):1120-1130. doi:10.1097/AOG.0000000000003267

6. Sieberg CB, Lunde CE, Borsook D. Endometriosis and pain in the adolescent- striking early to limit suffering: A narrative review. Neurosci Biobehav Rev. 2020;108:866-876. doi:10.1016/j. neubiorev.2019.12.004

7. As-Sanie S, Kim J, Schmidt-Wilcke T, et al. Functional connectivity is associated with altered brain chemistry in women with endometriosis-associated chronic pelvic pain. J Pain. 2016;17:1-13. Churchill Livingstone Inc. doi:10.1016/j.jpain.2015.09.008

8. As-Sanie S, Harris RE, Napadow V, et al. Changes in regional gray matter volume in women with chronic pelvic pain: A voxel-based morphometry study. Pain. 2012;153(5):1006-1014. doi:10.1016/j. pain.2012.01.032

9. Berkley KJ, Rapkin AJ, Papka RE. The pains of endometriosis. Science. 2005;308(5728):1587-1589. doi:10.1126/science.1111445

10. Dowlut-McElroy T, Strickland JL. Endometriosis in adolescents. Curr Opin Obstet Gynecol. 2017;29(5):306-309. doi:10.1097/ GCO.0000000000000402

11. Brosens I, Gargett CE, Guo SW, et al. Origins and progression of adolescent endometriosis. Reprod Sci. 2016;23(10):1282-1288. doi:10.1177/1933719116637919

12. Lou BM. Big picture of endometriosis helps provide guidance on approach to teens: comparative historical data show endo starting younger, is more severe. J Pediatr Adolesc Gynecol. 2003;16 (3SUPPL). doi:10.1016/S1083-3188(03)00063-9

13. Nahman-Averbuch H, Martucci KT, Granovsky Y, Weissman-Fogel I, Yarnitsky D, Coghill RC. Distinct brain mechanisms support spatial vs temporal filtering of nociceptive information. Pain. 2014;155 (12):2491-2501. doi:10.1016/j.pain.2014.07.008

14. Grill JD, Coghill RC. Transient analgesia evoked by noxious stimulus offset. $J$ Neurophysiol. 2002;87(4):2205-2208. doi:10.1152/ jn.00730.2001

15. Hermans L, Calders P, Van Oosterwijck J, Verschelde E, Bertel E, Meeus M. Systematic review an overview of offset analgesia and the comparison with conditioned pain modulation: a systematic literature review. Pain Physician. 2016;9:307-326.

16. Martucci KT, Eisenach JC, Tong C, Coghill RC. Opioid-independent mechanisms supporting offset analgesia and temporal sharpening of nociceptive information. Pain. 2012;153(6):1232-1243. doi:10.1016/ j.pain.2012.02.035

17. Niesters M, Hoitsma E, Sarton E, Aarts L, Dahan A. Offset analgesia in neuropathic pain patients and effect of treatment with morphine and ketamine. Anesthesiology. 2011;115(5):1063-1071. doi:10.1097/ ALN.0b013e31822fd03a

18. Petersen KK, Simonsen O, Olesen AE, Mørch CD, Arendt-Nielsen L. Pain inhibitory mechanisms and response to weak analgesics in patients with knee osteoarthritis. Eur $J$ Pain. 2019;23 (10):1904-1912. doi:10.1002/ejp.1465

19. Niesters M, Proto P, Aarts L, Sarton E, Dahan A. Tapentadol potentiates descending pain inhibition in chronic pain patients with diabetic polyneuropathy - pubMed. Br J Anaesth. 2014;113(1):148-156. doi:10.1093/bja/aeu056

20. Suzan E, Treister R, Pud D, Haddad M, Eisenberg E. The effect of hydromorphone therapy on psychophysical measurements of the descending inhibitory pain systems in patients with chronic radicular pain. Pain Med. 2015;16(1):168-175. doi:10.1111/pme.12565 
21. Rolke R, Baron R, Maier C, et al. Quantitative sensory testing in the German Research Network on Neuropathic Pain (DFNS): standardized protocol and reference values. Pain. 2006;123(3):231-243. doi:10.1016/j.pain.2006.01.041

22. Tham SW, Palermo TM, Holley AL, et al. A population-based study of quantitative sensory testing in adolescents with and without chronic pain. Pain. 2016;157(12):2807-2815. doi:10.1097/j.pain. 0000000000000716

23. Sethna NF, Meier PM, Zurakowski D, Berde CB. Cutaneous sensory abnormalities in children and adolescents with complex regional pain syndromes. Pain. 2007;131(1-2):153-161. doi:10.1016/j.pain.2006. 12.028

24. Zohsel K, Hohmeister J, Oelkers-Ax R, Flor H, Hermann C. Quantitative sensory testing in children with migraine: preliminary evidence for enhanced sensitivity to painful stimuli especially in girls. Pain. 2006;123(1-2):10-18. doi:10.1016/j.pain.2005.12.015

25. Duarte MA, Goulart EMA, Penna FJ. Pressure pain threshold in children with recurrent abdominal pain. J Pediatr Gastroenterol Nutr. 2000;31(3):280-285. doi:10.1097/00005176-200009000-00015

26. Brandow AM, Stucky CL, Hillery CA, Hoffmann RG, Panepinto JA. Patients with sickle cell disease have increased sensitivity to cold and heat. Am J Hematol. 2013;88(1):37-43. doi:10.1002/ajh.23341

27. O'Leary JD. Thermal pain and sensory processing in children with sickle cell disease. Clin J Pain. 2014;30(3):244-250. doi:10.1097/ AJP.0b013e318292a38e

28. King CD, Jastrowski Mano KE, Barnett KA, Pfeiffer M, Ting TV, Kashikar-Zuck S. Pressure pain threshold and anxiety in adolescent females with and without juvenile fibromyalgia. Clin J Pain. 2017;33 (7):620-626. doi:10.1097/AJP.0000000000000444

29. Cornelissen L, Donado C, Kim J, et al. Pain hypersensitivity in juvenile idiopathic arthritis: A quantitative sensory testing study. Pediatr Rheumatol. 2014;12(1). doi:10.1186/1546-0096-12-39

30. Blankenburg M, Junker J, Hirschfeld G, et al. Quantitative sensory testing profiles in children, adolescents and young adults (6-20 years) with cerebral palsy: hints for a neuropathic genesis of pain syndromes. Eur J Paediatr Neurol. 2018;22(3):470-481. doi:10.1016/j. ejpn.2017.12.015

31. Brawn J, Morotti M, Zondervan KT, Becker CM, Vincent K. Central changes associated with chronic pelvic pain and endometriosis. Hum Reprod Update. 2014;20(5):737-747. doi:10.1093/humupd/dmu025

32. Szikszay TM, Adamczyk WM, Luedtke K. The magnitude of offset analgesia as a measure of endogenous pain modulation in healthy participants and patients with chronic pain: A systematic review and meta-analysis. Clin J Pain. 2019;35(2):189-204. doi:10.1097/ AJP.0000000000000657

33. Yelle MD, Oshiro Y, Kraft RA, Coghill RC. Temporal filtering of nociceptive information by dynamic activation of endogenous pain modulatory systems. $J$ Neurosci. 2009;29(33):10264-10271. doi:10.1523/JNEUROSCI.4648-08.2009

34. Yelle MD, Rogers JM, Coghill RC. Offset analgesia: a temporal contrast mechanism for nociceptive information. Pain. 2008;134(1-2):174-186. doi:10.1016/j.pain.2007.04.014

35. Derbyshire SWG, Osborn J. Offset analgesia is mediated by activation in the region of the periaqueductal grey and rostral ventromedial medulla. Neuroimage. 2009;47(3):1002-1006. doi:10.1016/j. neuroimage.2009.04.032
36. Zhang S, Li T, Kobinata H, Ikeda E, Ota T, Kurata J. Attenuation of offset analgesia is associated with suppression of descending pain modulatory and reward systems in patients with chronic pain. Mol Pain. 2018;14:174480691876751. doi:10.1177/1744806918767512

37. Shulman J, Zurakowski D, Keysor J, Jervis K, Sethna NF. Offset analgesia identifies impaired endogenous pain modulation in pediatric chronic pain disorders. Pain. 2020. doi:10.1097/j.pain. 0000000000001984

38. Oudejans LCJ, Smit JM, Van Velzen M, Dahan A, Niesters M. The influence of offset analgesia on the onset and offset of pain in patients with fibromyalgia. Pain. 2015;156(12):2521-2527. doi:10.1097/j. pain.0000000000000321

39. Szikszay TM, Adamczyk WM, Carvalho GF, May A, Luedtke K. Offset analgesia: somatotopic endogenous pain modulation in migraine. Pain. 2020;161(3):557-564. doi:10.1097/j.pain.00 00000000001739

40. Kobinata H, Ikeda E, Zhang S, Li T, Makita K, Kurata J. Disrupted offset analgesia distinguishes patients with chronic pain from healthy controls. Pain. 2017;158(10):1951-1959. doi:10.1097/j.pain.0000000000000989

41. van Aken M, Oosterman J, van Rijn T, et al. Experimental pain tolerance is decreased and independent of clinical pain intensity in patients with endometriosis. Fertil Steril. 2018;110(6):1118-1128. doi:10.1016/j.fertnstert.2018.06.040

42. Hellman KM, Roth GE, Dillane KE, et al. Dysmenorrhea subtypes exhibit differential quantitative sensory assessment profiles. Pain. 2020;161(6):1227-1236. doi:10.1097/j.pain.0000000000001826

43. Jarrett ME, Shulman RJ, Cain KC, et al. Conditioned pain modulation in women with irritable bowel syndrome. Biol Res Nurs. 2014;16 (4):368-377. doi:10.1177/1099800413520486

44. Grinberg K, Granot M, Lowenstein L, Abramov L, Weissman-Fogel I. A common pronociceptive pain modulation profile typifying subgroups of chronic pelvic pain syndromes is interrelated with enhanced clinical pain. Pain. 2017;158(6):1021-1029. doi:10.1097/ j.pain.0000000000000869

45. Palit S, Bartley EJ, Kuhn BL, et al. Endogenous inhibition of pain and spinal nociception in women with premenstrual dysphoric disorder. J Pain Res. 2016;9:57-66. doi:10.2147/JPR.S97109

46. Pogatzki-Zahn EM, Drescher C, Englbrecht JS, Klein T, Magerl W, Zahn PK. Progesterone relates to enhanced incisional acute pain and pinprick hyperalgesia in the luteal phase of female volunteers. Pain. 2019;160(8):1781-1793. doi:10.1097/j.pain.0000000000001561

47. Okifuji A, Turk DC. Sex hormones and pain in regularly menstruating women with fibromyalgia syndrome. $J$ Pain. 2006;7 (11):851-859. doi:10.1016/j.jpain.2006.04.005

48. Stening K, Eriksson O, Wahren LK, Berg G, Hammar M, Blomqvist A. Pain sensations to the cold pressor test in normally menstruating women: comparison with men and relation to menstrual phase and serum sex steroid levels. Am J Physiol Regul Integr Comp Physiol. 2007;293(4):R1711-R1716. doi:10.1152/ajpregu.00127. 2007

49. Kowalczyk WJ, Evans SM, Bisaga AM, Sullivan MA, Comer SD. Sex differences and hormonal influences on response to cold pressor pain in humans. J Pain. 2006;7(3):151-160. doi:10.1016/j.jpain.2005.10.004

50. Simons LE, Elman I, Borsook D. Psychological processing in chronic pain: A neural systems approach. Neurosci Biobehav Rev. 2014;39:61-78. doi:10.1016/j.neubiorev.2013.12.006 


\section{Publish your work in this journal}

The Journal of Pain Research is an international, peer reviewed, open access, online journal that welcomes laboratory and clinical findings in the fields of pain research and the prevention and management of pain. Original research, reviews, symposium reports, hypothesis formation and commentaries are all considered for publication. The manuscript management system is completely online and includes a very quick and fair peer-review system, which is all easy to use. Visit http:// www.dovepress.com/testimonials.php to read real quotes from published authors. 